\title{
Akt regulates $\beta$-catenin in a rat model of focal cerebral ischemia-reperfusion injury
}

\author{
XUE-SONG XING ${ }^{1,2}$, FANG LIU ${ }^{1}$ and ZHI-YI HE ${ }^{2}$ \\ ${ }^{1}$ Department of Neurology, Shenyang Medical College, Fengtian Hospital, Shenyang, Liaoning 110024; \\ ${ }^{2}$ Department of Neurology, The First Affiliated Hospital, China Medical University, Shenyang, Liaoning 110001, P.R. China
}

Received February 11, 2014; Accepted November 3, 2014

DOI: $10.3892 / \mathrm{mmr} .2014 .3000$

\begin{abstract}
The present study aimed to investigate the effects of the phosphoinositide 3-kinase (PI3K)/Akt signaling pathway on the $\mathrm{Wnt} / \beta$-catenin signaling pathway in rats with focal cerebral ischemia-reperfusion injury. A total of 96 rat focal cerebral ischemia-reperfusion models, established according to a modified version of Longa's method, were randomly divided into four groups: Sham-operated (S), cerebral ischemia-reperfusion injury (I), cerebral ischemia-reperfusion + basic fibroblast growth factor (bFGF) post-processing and, finally, cerebral ischemia-reperfusion + bFGF post-processing + PI3K inhibitor LY294002 (LY). Each group consisted of 24 rats and each group was divided into four subgroups according to the indicated reperfusion times of $12,24,48$ and $72 \mathrm{~h}$. The morphological changes of the cortical tissue and the cellular apoptosis were determined using hematoxylin and eosin staining and the terminal deoxynucleotidyl transferase dUTP nick end labeling method, respectively. The expression levels of phosphory-

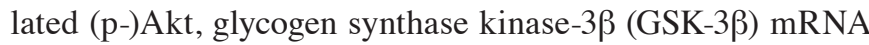
and $\beta$-catenin in the cortical tissue were detected at different time-points. The number of apoptotic cells and the expression levels of p-Akt, GSK-3 $\beta$ mRNA and $\beta$-catenin in the I and LY groups were significantly higher compared with those in the $S$ group $(\mathrm{P}<0.05)$. In the bFGF group, the number of apoptotic cells and the mRNA expression levels of GSK-3 $\beta$ were significantly decreased, whereas the expression levels of p-Akt and $\beta$-catenin were significantly increased compared with those in the I and LY groups $(\mathrm{P}<0.05)$. In cerebral ischemia-reperfusion injury, the PI3K/Akt signaling pathway regulated $\beta$-catenin, the main member of the Wnt signaling pathway, via GSK-3 $\beta$, providing information to assist in further investigation of the mechanism of $\beta$-catenin in ischemia-reperfusion injury.
\end{abstract}

Correspondence to: Dr Zhi-Yi He, Department of Neurology, The First Affiliated Hospital, China Medical University, 155 Nanjing Street, Shenyang, Liaoning 110001, P.R. China

E-mail: zhiyihecn@163.com

Key words: cerebral ischemia, Akt, glycogen synthase kinase-3 $\beta$, $\beta$-catenin, basic fibroblast growth factor, LY294002

\section{Introduction}

Necrosis and apoptosis are two common and important patterns of cell death in ischemia-reperfusion injury. The process of nerve cell death cannot be stopped, unlike apoptosis, which is a programmed process $(1,2)$; however, apoptosis caused by ischemic brain injury can be prevented by interfering with the apoptotic process. Glycogen synthase kinase-3 $\beta$ (GSK-3 $\beta$ ) regulates the $\beta$-catenin/Wnt signaling pathway and functions as a downstream molecule of the phosphoinositide 3-kinase (PI3K)/Akt signaling pathway $(3,4)$. GSK-3 $\beta$, an active serine/threonine-protein kinase in cells, is widely involved in regulating basic cellular functions including cell cycle, gene expression and cytoskeleton stabilization via protein phosphorylation $(5,6)$. Abundant GSK-3 in the brain is also a negative regulatory factor of signaling cascades, including PI3K/Akt (7). GSK-3 $\beta$ can be deactivated through Akt-mediated phosphorylation at serine 9, which produces antiapoptotic effects $(8,9)$. During GSK-3 $\beta$ phosphorylation, $\beta$-catenin dissociates from the anaphase-promoting complex, enters the nucleus and potentiates the transcription of Wnt target genes, thereby promoting cell proliferation and inhibiting apoptosis (10-12). Previous studies on the regulation of $\beta$-catenin by Akt have focussed on tumor prevention; however, the applicability of this regulation in ischemic brain injury remains to be elucidated.

In the present study, basic fibroblast growth factor (bFGF) and the PI3K inhibitor LY294002 were administered in a rat model in vivo to activate and inhibit Akt activity, respectively. The intracellular $\beta$-catenin content was also examined to investigate the regulatory effects of Akt on $\beta$-catenin and to further elucidate the mechanism of $\beta$-catenin in ischemic brain injury.

\section{Materials and methods}

Experimental animals. A total of 96 healthy male Sprague Dawley rats aged between 10 and 12 weeks (280-320 g) were provided by the Laboratory Animal Center of Shenyang Medical College [Shenyang, China; animal certification no. SCXK (liao) 2003-0016]. Animals were kept at $25-30^{\circ} \mathrm{C}$ in $60-70 \%$ relative humidity, with a 12 hour light:dark cycle and ad libitum access to food and water. The rat models of focal cerebral ischemia-reperfusion were established according to 
the modified Longa's (13) method and were randomly divided into four groups with 24 rats in each group. These groups were labeled as: The sham-operated (S), cerebral ischemia-reperfusion injury (I), cerebral ischemia-reperfusion + bFGF post-processing (bFGF) and cerebral ischemia-reperfusion + bFGF post-processing + LY294002 (LY) groups. Each group was further divided into four subgroups, according to different reperfusion times of $12,24,48$ and $72 \mathrm{~h}$. The present study was performed in accordance with the recommendations in the Guide for the Care and Use of Laboratory Animals of the National Institutes of Health. The animal use procedures were reviewed and approved by the Institutional Animal Care and Use Committee of the First Affiliated Hospital, China Medical University (Shenyang, China).

Animal models. The focal cerebral ischemia-reperfusion injury was induced in the rats according to a modification of Longa's method (14). Anesthesia was induced via intraperitoneal injection of $10 \%$ chloral hydrate $(350 \mathrm{mg} / \mathrm{kg}$; Sigma-Aldrich, St. Louis, MO, USA). A median cervical incision was then performed to expose the right common carotid artery (CCA), external carotid artery (ECA) and internal carotid artery (ICA). Following ligation of the CCA, ECA and ICA, a 4-0 nylon suture with a rounded end was inserted into the ICA $2 \mathrm{~mm}$ above the bifurcation of the CCA until a slight resistance was felt. The insertion depth of the suture was between 18 and $20 \mathrm{~mm}$. For the sham-operated rats, the insertion depth was $10 \mathrm{~mm}$. After $2 \mathrm{~h}$ occlusion, reperfusion was permitted by drawing the nylon suture. The room temperature was maintained between 25 and $28^{\circ} \mathrm{C}$ during and following the surgery. The rectal temperature of the rats was maintained at $37.0 \pm 0.5^{\circ} \mathrm{C}$ by using incandescent lamps. The focal cerebral ischemia-reperfusion injury was successfully induced when the conscious rats exhibited hemiplegia on the left side of their bodies, specifically on the left forelimb. Subsequently, $30 \mathrm{~min}$ after the induction of ischemia-reperfusion injury, $10 \mu \mathrm{l}$ physiological saline containing $1.2 \mu \mathrm{g}$ bFGF (Sigma-Aldrich), was administered via the right lateral ventricle. At each indicated time-point, $20 \mu \mathrm{l}$ physiological saline was injected into the right lateral ventricle of the rats in the $\mathrm{S}$ and I groups. At $15 \mathrm{~min}$ prior to reperfusion, $0.3 \mathrm{mg} / \mathrm{kg}$ LY294002 (Cell Signaling Technology, Inc., Danvers, MA, USA) was injected via the femoral vein. The rats from each group were then re-anesthetized and administered with $4 \%$ paraformaldehyde via cardiac perfusion at different reperfusion time-points $(12,24,48$ and 72 h). The whole brain was harvested and fixed for the preparation of $5 \mu \mathrm{m}$ paraffin (Sigma-Aldrich) sections, which were stained with hematoxylin and eosin (Sigma-Aldrich).

Terminal deoxynucleotidyl transferase dUTP nick end labeling (TUNEL). An in situ end-labeling apoptosis detection kit (Haoyang Biological Product Science and Technology Co. Ltd., Tianjin, China) was used according to the manufacturer's instructions. The paraffin sections were routinely dehydrated in a series of graded ethanol solutions and treated with $3 \% \mathrm{H}_{2} \mathrm{O}_{2}$ (Zhongshan Gold Bridge Biotechnology Co., Ltd., Beijing, China), for $10 \mathrm{~min}$ to deactivate endogenous peroxidase. The sections were then subjected to microwave antigen retrieval (Zhongshan Gold Bridge Biotechnology Co., Ltd.) and incubated at $37^{\circ} \mathrm{C}$ for
$1 \mathrm{~h}$ following the addition of TUNEL solution and at $37^{\circ} \mathrm{C}$ for $30 \mathrm{~min}$ following the addition of propylene oxide (Zhongshan Gold Bridge Biotechnology Co., Ltd.). Finally, the sections were developed using diaminobenzidine (Zhongshan Gold Bridge Biotechnology Co., Ltd.), dehydrated, cleared and mounted. Sections were viewed using an Olympus DSX500 (Wuxi Lianfa Technology Co., Ltd., Wuxi, China).

Immunohistochemical staining. The streptavidin-peroxidase method was used for immunohistochemical analysis. The paraffin sections were dehydrated in a series of graded ethanol (Zhongshan Gold Bridge Biotechnology Co., Ltd.) solutions, treated with $3 \% \mathrm{H}_{2} \mathrm{O}_{2}$ at room temperature for $10 \mathrm{~min}$, subjected to microwave antigen retrieval, inhibited using $50 \mu \mathrm{l}$ goat serum (Zhongshan Gold Bridge Biotechnology Co., Ltd.) and incubated at room temperature for $20 \mathrm{~min}$. Following the addition of phosphorylated (p-)Akt primary antibody (1:200; Cell Signaling Technology, Inc.), the sections were incubated at $4^{\circ} \mathrm{C}$ overnight. Biotinylated secondary working solution (Zhongshan Gold Bridge Biotechnology Co. Ltd., Beijing, China) was then added and incubated at $37^{\circ} \mathrm{C}$ for $30 \mathrm{~min}$, followed by addition of horseradish peroxidase (HRP)-conjugated streptavidin (Zhongshan Gold Bridge Biotechnology Co., Ltd.) working solution and incubation at $37^{\circ} \mathrm{C}$ for $30 \mathrm{~min}$. The sections were developed using diaminobenzidine and were then routinely dehydrated, cleared and mounted. Phosphate-buffered saline (PBS; Zhongshan Gold Bridge Biotechnology Co., Ltd.) was used instead of the primary antibody in the negative control.

In situ hybridization. The present study used digoxin-labeled multiphase oligonucleotide probes (Zhongshan Gold Bridge Biotechnology Co., Ltd.) and the sequence of GSK-3 $\beta$ was 5'-CTCCTCGGACCAGCTGCTTTGCACTTCCAA-3'. The sections were treated with freshly prepared $0.5 \% \mathrm{H}_{2} \mathrm{O}_{2}$ in methanol at room temperature for $30 \mathrm{~min}$ and were digested with pepsin (Zhongshan Gold Bridge Biotechnology Co., Ltd.), which was freshly diluted with $3 \%$ citric acid. Subsequently, The sections were pre-hybridized for $2 \mathrm{~h}$ in a thermostat container at $37^{\circ} \mathrm{C}$ and, following addition of the hybridization solution, were incubated overnight at $37^{\circ} \mathrm{C}$. The sections were then treated with blocking solution, biotinylated rat anti-digoxin, streptavidin-peroxidase complex and biotinylated peroxidase (Zhongshan Gold Bridge Biotechnology Co., Ltd.). Finally, the sections were routinely dehydrated, cleared and mounted. PBS was used as the hybridization solution in the negative control.

Western blot analysis. At each indicated time-point following intraperitoneal injection of $10 \%$ chloral hydrate $(350 \mathrm{mg} / \mathrm{kg})$, the brain of each rat was harvested and placed on ice; the cortical tissues were stored at $-70^{\circ} \mathrm{C}$ for later use. Following addition of the homogenate $\left(1: 9 ; \mathrm{NaH}_{2} \mathrm{PO}_{4}\right.$ $\left.\left(\mathrm{H}_{2} \mathrm{O}\right), \mathrm{NaH}_{2} \mathrm{PO}_{4} \cdot 12 \mathrm{H}_{2} \mathrm{O}, \mathrm{NaCl}\right)$, the samples were lysed and homogenated on ice. The supernatant was collected and protein levels were determined using the Coomassie Brilliant blue G-250 method (15). Equal quantities of lysate protein were run on $10 \%$ SDS-PAGE gels and electrophoretically transferred onto a polyvinylidene difluoride membrane (Zhongshan Gold Bridge Biotechnology Co., Ltd.). Following 


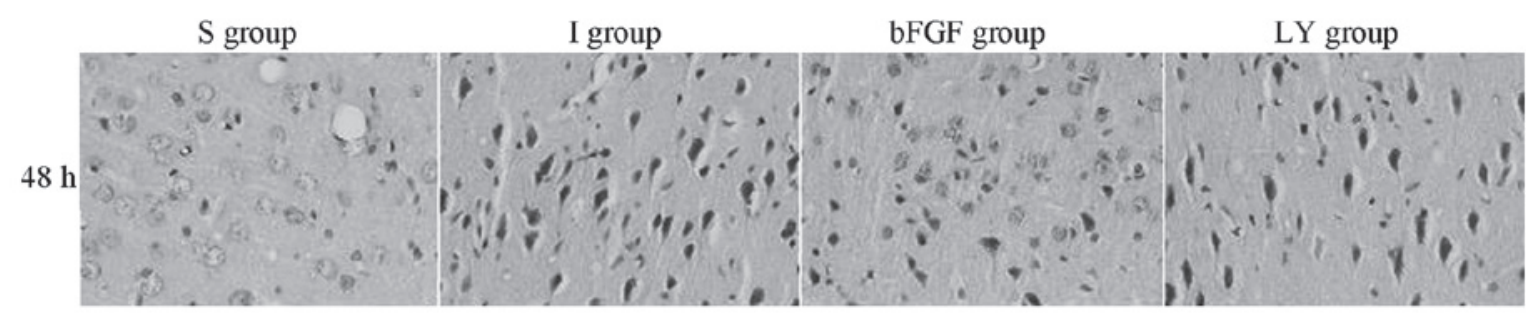

Figure 1. Hematoxylin and eosin staining for the detection of histological changes in the cortex following ischemia-reperfusion injury. The S group exhibited normal nerve cells. Histological changes were observed in the right cortex in the I and LY groups after $48 \mathrm{~h}$ and these histological changes were partially alleviated in the bFGF group. Magnification, $x 400$. S, sham-operated group; I, cerebral ischemia-reperfusion injury group; bEGF, cerebral ischemia-reperfusion + bFGF post-processing; LY, cerebral ischemia-reperfusion + bFGF post-processing + LY294002; bFGF, basic fibroblast growth factor.

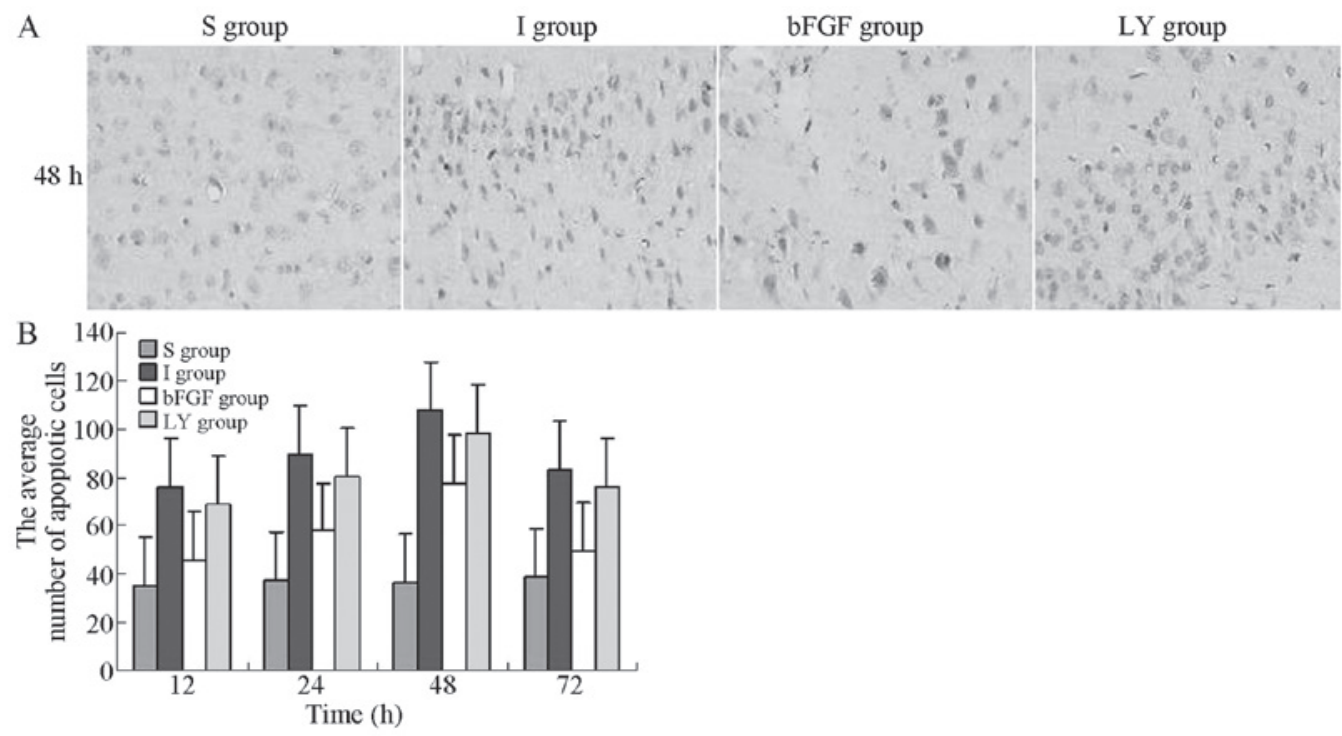

Figure 2. TUNEL detection of apoptotic cells in the right cortex following ischemia-reperfusion injury in each group. TUNEL-positive cells exhibited brown granules in the nuclei. Magnification, x400. (A) Number of apoptotic cells in the right cortex 12, 24, 48 and $72 \mathrm{~h}$ after ischemia-reperfusion injury. (B) Average number of apoptotic cells $48 \mathrm{~h}$ after ischemia-reperfusion injury. The average number of apoptotic cells in the right cortex at each indicated time-point is expressed as the mean \pm standard deviation ( $\mathrm{n}=6$ for each time-point). $\mathrm{P}<0.05$, I group or LY group, vs. $\mathrm{S}$ group; $\mathrm{P}<0.05$, bFGF group, vs. I group or LY group. S, sham-operated; I, cerebral ischemia-reperfusion injury; bEGF, cerebral ischemia-reperfusion + bFGF post-processing; LY, cerebral ischemia-reperfusion + bFGF post-processing + LY294002; TUNEL, terminal deoxynucleotidyl transferase dUTP nick end labeling; bFGF, basic fibroblast growth factor.

inhibition with 5\% defatted milk powder (Sigma-Aldrich), the blots were incubated overnight at $4^{\circ} \mathrm{C}$. They were then incubated for $2 \mathrm{~h}$ at room temperature with the $\beta$-catenin primary antibody (1:200) and for $1 \mathrm{~h}$ at room temperature with the HRP-conjugated secondary antibody (1:200). Enhanced chemiluminescence (ECL; Sigma-Aldrich) was used for the visualization of the protein bands.

Statistical analysis. The average number of apoptotic cells was determined using a MetaMorph/Evolution MP5.0 micro-imaging analysissystem(Alpha,USA).Theaverageoptical densities of the cortical p-Akt and cortical GSK-3 $\beta$ mRNA and the integrated density value of the cortical $\beta$-catenin band were determined by immunohistochemical staining, in situ hybridization and western blot analysis, respectively. All data were statistically analyzed using SPSS 13.0 software (SPSS, Inc., Chicago, IL, USA) and are expressed as the mean \pm standard deviation. Analysis of variance was used to assess the difference between the means. $\mathrm{P}<0.05$ was considered to indicate a statistically significant difference.

\section{Results}

bFGF improves survival of neuronal cells following ischemia-reperfusion via activating the PI3K/Akt signaling pathway. The histological changes in the right cortex, caused by the cerebral ischemia-reperfusion injury, were evaluated under an optical microscope (Fig. 1). The S group exhibited a large number of orderly arranged neurons with complete morphology. In the I and LY groups, the neurons became plump and were distributed unevenly, with widened cellular interspaces. Several cytons became small and the nuclei were pyknotic and darkly stained. All of these characteristics were most marked after $48 \mathrm{~h}$. In the bFGF group, increased neuron survival was observed in the right cortex compared with that in the I and LY groups, cyton swelling was alleviated and cell morphology was significantly improved. bFGF promoted the survival of the nerve cells in the ischemic cortex inhibited by LY294002, a PI3K/Akt signaling pathway-specific inhibitor. These findings suggested that bFGF activated the PI3K/Akt signaling pathway. 

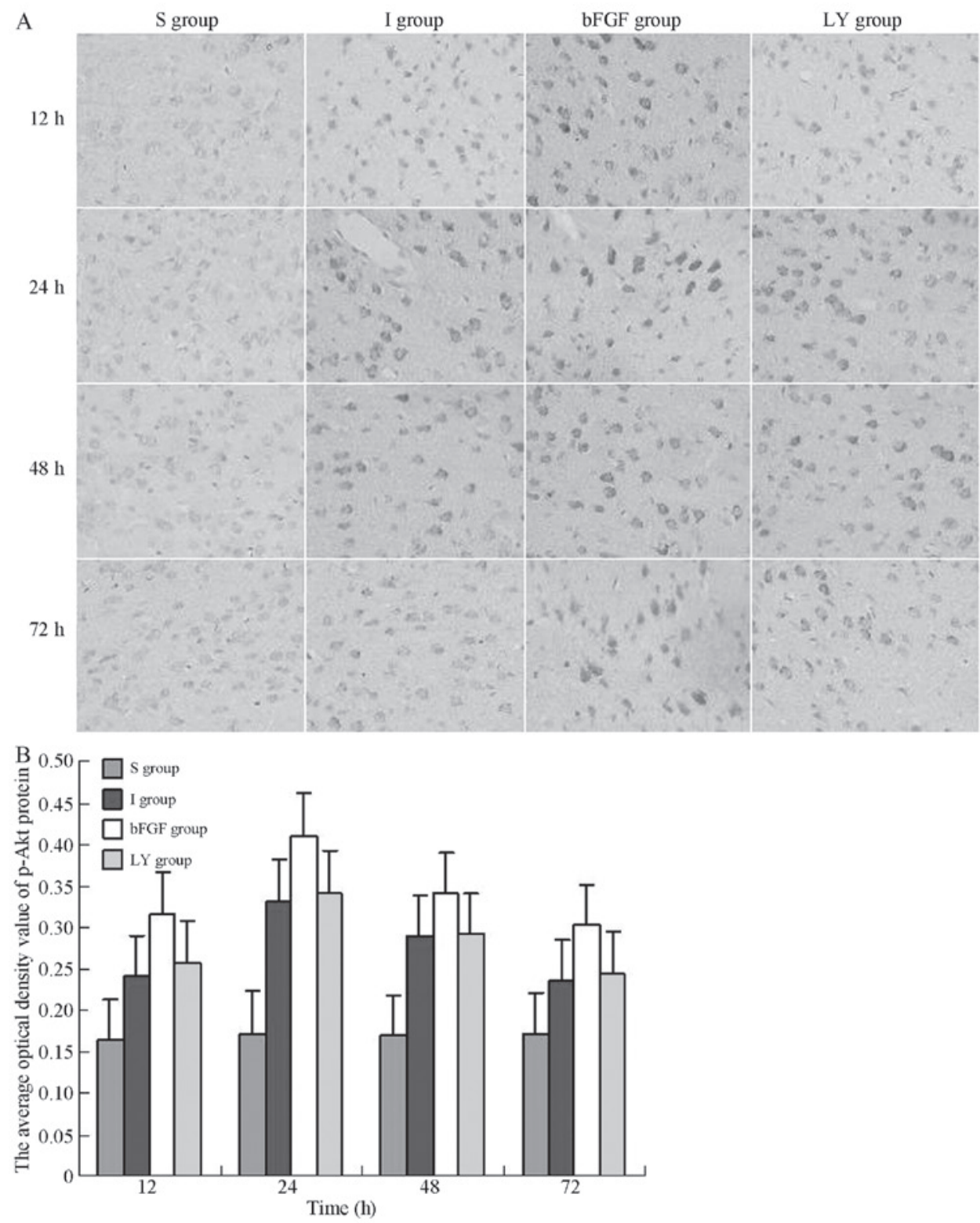

Figure 3. Immunohistochemical assessment of the cortical expression of p-Akt following ischemia-reperfusion injury. p-Akt immunoreactive cells exhibited brown granules in the cytoplasm and nuclei. Magnification, x400. (A) p-Akt-positive protein in the right cortex 12, 24, 48 and $72 \mathrm{~h}$ after ischemia-reperfusion injury. (B) Average optical density values of p-Akt-positive protein in each group. All data are expressed as the mean \pm standard deviation ( $\mathrm{n}=6$ rats for each time-point). $\mathrm{P}<0.05$, I group or LY group, vs $\mathrm{S}$ group; $\mathrm{P}<0.05$, bFGF group, vs. I group or LY group. $\mathrm{S}$, sham-operated; I, cerebral ischemia-reperfusion injury; bEGF, cerebral ischemia-reperfusion + bFGF post-processing; LY, cerebral ischemia-reperfusion + bFGF post-processing + LY294002; p-, phosphorylated; bFGF, basic fibroblast growth factor.

bFGF inhibits apoptosis of neuronal cells following ischemia-reperfusion via activating the PI3K/Akt signaling pathway. The apoptosis of nerve cells in the right cortex, induced by cerebral ischemia-reperfusion injury, was evaluated under an optical microscope. The TUNEL-positive cells exhibited yellow-brown granules in the nuclei. In the I and LY groups, the number of apoptotic cells gradually increased with reperfusion time, reaching a peak level at $48 \mathrm{~h}$, followed by a gradual decrease after $72 \mathrm{~h}$. At the same indicated time-points, the numbers of apoptotic cells in the I and LY groups were increased significantly compared with those in the $\mathrm{S}$ group $(\mathrm{P}<0.05)$, whereas the numbers of apoptotic cells in the bFGF group were significantly lower compared with those in the I and LY groups
( $\mathrm{P}<0.05$; Fig. 2). bFGF inhibited the apoptosis of nerve cells in the ischemic cortex and the effect of bFGF was inhibited by LY294002. This observation suggested that bFGF inhibited cellular apoptosis by activating the PI3K/Akt signaling pathway.

bFGF activates cortical Akt following ischemia-reperfusion injury. Following ischemia-reperfusion injury, the expression of cortical p-Akt was investigated at each time-point by immunohistochemical staining in each group to determine whether ischemia-reperfusion injury and bFGF activated Akt. In the $\mathrm{S}$ group, a marginal expression of $\mathrm{p}-\mathrm{Akt}$ was observed in the cortex. In the I and LY groups, the expression of p-Akt appeared in the right cortex at $12 \mathrm{~h}$, peaked at $24 \mathrm{~h}$ and gradually 

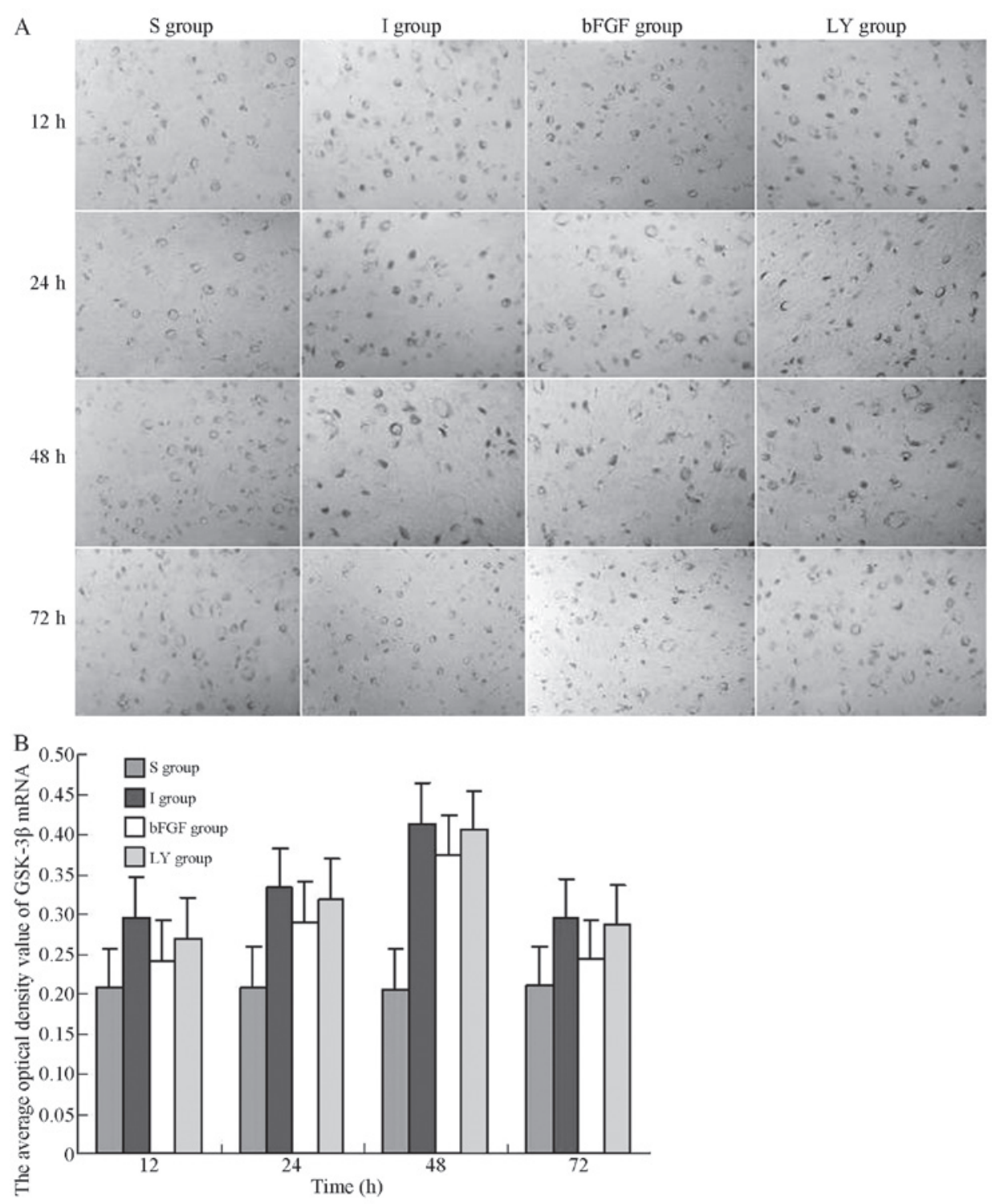

Figure 4. In situ hybridization for the detection of the mRNA expression of GSK-3 $\beta$ in the right cortex following ischemia-reperfusion injury. Magnification, $\mathrm{x} 400$. (A) mRNA expression levels of GSK-3 $\beta$ in the right cortex 12, 24, 48, $72 \mathrm{~h}$ after ischemia-reperfusion injury. (B) Average optical density values of GSK-3 $\beta$ mRNA-positive product in each group. All data are expressed as the mean \pm standard deviation $(n=6$ rats for each time-point). $P<0.05$, I group or LY group, vs. S group; P<0.05, bFGF group, vs. I group or LY group. S, sham-operated; I, cerebral ischemia-reperfusion injury; bEGF, cerebral ischemia-reperfusion + bFGF post-processing; LY, cerebral ischemia-reperfusion + bFGF post-processing + LY294002; GSK-3 $\beta$, glycogen synthase kinase-3 $\beta$; bFGF, basic fibroblast growth factor.

decreased following this. Of note, the expression of p-Akt in the groups that underwent ischemia-reperfusion remained significantly higher compared with that in the $\mathrm{S}$ group $(\mathrm{P}<0.05$; Fig. 3). In the bFGF group, the expression of p-Akt exhibited a similar trend; however, the expression levels were significantly higher compared with those in the I and LY groups $(\mathrm{P}<0.05$; Fig. 3). These findings suggested that bFGF activated Akt in ischemic brain injury and that the effect of bFGF was inhibited by LY294002, an inhibitor of the PI3K/Akt signaling pathway.

bFGF suppresses cortical mRNA expression of GSK-3 $\beta$ following ischemia-reperfusion injury. To investigate whether ischemia-reperfusion injury and bFGF activated GSK-3 $\beta$, the mRNA expression levels of GSK-3 $\beta$ at each indicated time-point following ischemia-reperfusion injury were investigated. The in situ hybridization assay indicated that GSK- $3 \beta$ mRNA was expressed in the right cortex in the I and LY groups; the mRNA expression of GSK-3 $\beta$ appeared $12 \mathrm{~h}$ after reperfusion, peaked at $48 \mathrm{~h}$ and decreased after $72 \mathrm{~h}$. Of note, the GSK-3 $\beta$ expression levels in the I and LY groups were higher compared with those in the $\mathrm{S}$ group $(\mathrm{P}<0.05$; Fig. 4). The mRNA expression of GSK-3 $\beta$ in the right cortex was significantly decreased in the bFGF group compared with those in the I and LY groups $(\mathrm{P}<0.05$; Fig. 4). bFGF inhibited the mRNA expression of GSK-3 $\beta$ in the cortex subjected to ischemia-reperfusion injury. LY294002, the PI3 K/Akt pathway inhibitor, upregulated the mRNA expression of GSK- $3 \beta$ and effectively inhibited the effect of bFGF.

bFGF promotes cortical $\beta$-catenin expression following ischemia-reperfusion injury. Western blot analysis was 

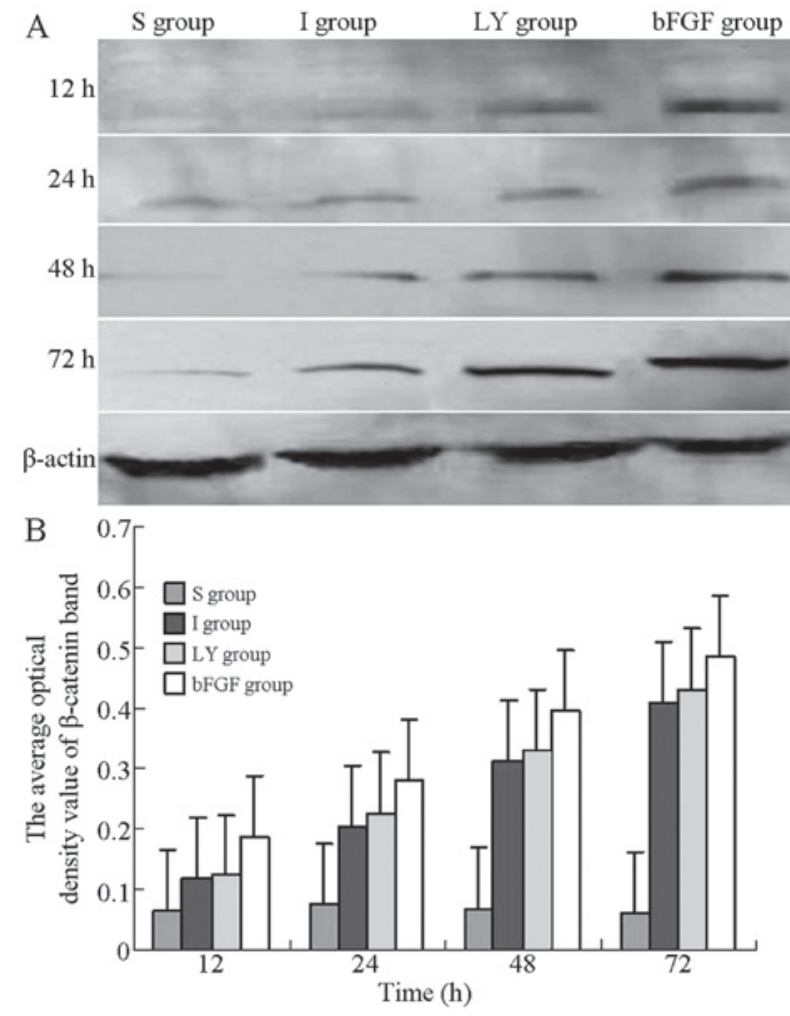

Figure 5. Western blot analysis for detection of the expression of $\beta$-catenin in the right cortex following ischemia-reperfusion injury. (A) Protein expression of $\beta$-catenin in the right cortex $12,24,48$ and $72 \mathrm{~h}$ after ischemia-reperfusion injury. (B) Optical density values of the $\beta$-catenin band at each time-point of reperfusion. All data are expressed as the mean \pm standard deviation $(n=6$ rats for each time-point). $\mathrm{P}<0.05$, I or LY group, vs. $\mathrm{S}$ group; $\mathrm{P}<0.05$, bFGF group, vs. I or LY group. S, sham-operated; I, cerebral ischemia-reperfusion injury; bEGF, cerebral ischemia-reperfusion + bFGF post-processing; LY cerebral ischemia-reperfusion + bFGF post-processing + LY294002; bFGF, basic fibroblast growth factor.

used to detect the expression of $\beta$-catenin at each time-point following ischemia-reperfusion injury and to evaluate the effects of bFGF and LY294002 on the expression of $\beta$-catenin. As Fig. 5A shows, the protein levels of $\beta$-catenin in the I and LY groups were reduced compared with those in the bFGF group, but higher compared with those in the $\mathrm{S}$ group. At 12, 24, 48 and $72 \mathrm{~h}$ after reperfusion, the optical densities of $\beta$-catenin in the I and LY groups were significantly higher compared with those in the $\mathrm{S}$ group, but were significantly lower compared with those in the bFGF group $(\mathrm{P}<0.05$; Fig. 5B). bFGF promoted the expression of $\beta$-catenin in the cortex subjected to ischemia-reperfusion injury; however, this promoting effect was inhibited by inhibition of the PI3K/Akt signaling pathway.

\section{Discussion}

$\beta$-catenin, a protein molecule with a relative molecular weight of 92,000 Da, was initially identified to co-precipitate with the E-cadherin cell-cell adhesive complex and was subsequently found to link E-cadherin to $\alpha$-catenin, which linked the E-cadherin/catenin complex to the cortical cytoskeleton $(16,17)$. Previous studies have suggested that $\beta$-catenin is also involved in Wnt signaling $(18,19)$. During Wnt signaling, $\beta$-catenin, without binding to E-cadherin in the cytoplasm, acts as a signal transduction and transcription factor, enters the nucleus, binds to $\mathrm{TCF} / \mathrm{LEF}$ and regulates the transcription of target genes. Thus, $\beta$-catenin is important in cell proliferation, embryonic development and tumor formation (20-22). Akt contains serine/threonine protein kinase and, following phosphorylation, Akt phosphorylates numerous intercellular substrate proteins and regulates their activities $(23,24)$. Therefore, Akt is involved in regulating a number of intercellular signal pathways, promoting cell survival and proliferation, preventing cellular apoptosis and regulating glycometabolism and protein synthesis (25-27).

bFGF, a polypeptide with various biological activities, functions in an identical manner as a neurotrophic factor (28-30) and promotes the survival of nerve cells via numerous conduction pathways. To the best of our knowledge, no previous studies have investigated whether bFGF can activate the Wnt/ $\beta$-catenin and PI3K/Akt signaling pathways simultaneously in ischemic brain injury. In the present study, LY294002, an inhibitor of the PI3K/Akt signaling pathway with high specificity and efficacy, was used to investigate the effects of inhibiting the PI3K/Akt signaling pathway and suppressing the expression of $\beta$-catenin, the main member of the Wnt/ $\beta$-catenin signaling pathway. Immunohistochemical staining results revealed that, at each indicated time-point, the protein expression levels of $\mathrm{p}-\mathrm{Akt}$ in the I and LY groups were higher compared with those in the $\mathrm{S}$ group and peaked at $24 \mathrm{~h}$. However, at each indicated time-point, the cortical expression levels of p-Akt in the bFGF group were higher compared with the levels in the I and LY groups. These findings suggested that, in ischemic brain injury, bFGF activated the PI3K/Akt signaling pathway and LY294002, as an inhibitor of the PI3K/Akt signaling pathway, effectively inhibited the bFGF-mediated activation of Akt. In situ hybridization and western blot analysis were also used to investigate the mRNA expression of GSK-3 $\beta$ and the expression of $\beta$-catenin in the ischemia-reperfusion cortex. The results demonstrated that, at each indicated time-point, the mRNA expression of GSK-3 $\beta$ and the expression of $\beta$-catenin in the I and LY groups were significantly higher compared with those in the $\mathrm{S}$ group and peaked at 48 and $72 \mathrm{~h}$, respectively. Compared with the I and LY groups, the mRNA expression of GSK-3 $\beta$ in the bFGF group was significantly decreased, whereas the protein expression of $\beta$-catenin was significantly increased at each time-point. These findings suggested that bFGF activated Akt, inhibited GSK-3 $\beta$ activity and antagonized the $\beta$-catenin-degradation complex, composed of GSK-3 $\beta$, axin and adenomatosis polyposis coli protein. In this process, $\beta$-catenin cannot be phosphorylated and the dissociated $\beta$-catenin accumulates in the cytoplasm, enters the nucleus, promotes Wnt target gene transcription and inhibits cellular apoptosis (31-33). When the PI3K/Akt signaling pathway was inhibited by LY294002, $\beta$-catenin was degraded by adenomatosis polyposis coli polyprotein and its expression in the ischemic cortex was reduced. The expression of Akt in the cortex peaked $24 \mathrm{~h}$ after ischemia-reperfusion injury and the expression of $\beta$-catenin increased continuously following ischemia-reperfusion and peaked after $72 \mathrm{~h}$. These findings suggested that $\beta$-catenin was involved in signal conduction following PI3K/Akt activation and the Akt regulation of $\beta$-catenin affected ischemic brain 
injury. In addition, Akt and $\beta$-catenin were activated by bFGF via the bFGF-Akt-GSK-3 $\beta-\beta$-catenin signaling cascade.

The present study established a rat model of focal cerebral ischemia-reperfusion injury, confirmed by histopathological changes and neuronal apoptosis using hematoxylin and eosin staining and the TUNEL method. In the bFGF group, few histological changes or apoptotic cells were observed in the ischemic cortex, whereas, the protective effect of bFGF was inhibited in the LY group. These findings suggested that the PI3K/Akt signaling pathway has a significant function in cell survival and that, by regulating $\beta$-catenin, the PI3K/Akt and Wnt signaling pathways exhibit protective effects on the brain. This observation indicates that $\mathrm{Akt}$ and $\beta$-catenin may be significant target molecules for the treatment of ischemic brain injury.

\section{Acknowledgements}

This study was supported by the Science Research Plan of Shenyang (no. F11-262-9-17).

\section{References}

1. Guo WP, Fu XG, Jiang SM and Wu JZ: Neuregulin-1 regulates the expression of Akt, Bcl-2, and Bad signaling after focal cerebral ischemia in rats. Biochem Cell Biol 88: 649-654, 2010.

2. Zhu J, Shen W, Gao L, et al: PI3K/Akt-independent negative regulation of JNK signaling by MKP-7 after cerebral ischemia in rat hippocampus. BMC Neurosci 14: 1, 2013.

3. Forde JE and Dale TC: Glycogen synthase kinase 3: a key regulator of cellular fate. Cell Mol Life Sci 64: 1930-1944, 2007.

4. Ye Z, Guo Q, Xia P, Wang N, Wang E and Yuan Y: Sevoflurane postconditioning involves an up-regulation of HIF- $1 \alpha$ and HO-1 expression via PI3K/Akt pathway in a rat model of focal cerebral ischemia. Brain Res 1463: 63-74, 2012.

5. Dugo L, Collin M and Thiemermann C: Glycogen synthase kinase 3 beta as a target for the therapy of shock and inflammation. Shock 27: 113-123, 2007.

6. Nishihara M, Miura T, Miki T, et al: Modulation of the mitochondrial permeability transition pore complex in GSK-3beta-mediated myocardial protection. J Mol Cell Cardiol 43: 564-570, 2007.

7. Zhao H, Sapolsky RM and Steinberg GK: Phosphoinositide-3-kinase/akt survival signal pathways are implicated in neuronal survival after stroke. Mol Neurobiol 34: 249-270, 2006.

8. Gao X, Zhang H, Takahashi T, et al: The Akt signaling pathway contributes to postconditioning's protection against stroke; the protection is associated with the MAPK and PKC pathways. J Neurochem 105: 943-955, 2008.

9. Peng B, Guo QL, He ZJ, et al: Remote ischemic postconditioning protects the brain from global cerebral ischemia/reperfusion injury by up-regulating endothelial nitric oxide synthase through the PI3K/Akt pathway. Brain Res 1445: 92-102, 2012.

10. Chong ZZ, Li F and Maiese K: Cellular demise and inflammatory microglial activation during beta-amyloid toxicity are governed by Wnt1 and canonical signaling pathways. Cell Signal 19: 1150-1162, 2007.

11. Murase S, Mosser E and Schuman EM: Depolarization drives beta-catenin into neuronal spines promoting changes in synaptic structure and function. Neuron 35: 91-105, 2002.

12. Zhang L, Zhang ZG, Liu XS, Hozeska-Solgot A and Chopp M: The PI3K/Akt pathway mediates the neuroprotective effect of atorvastatin in extending thrombolytic therapy after embolic stroke in the rat. Arterioscler Thromb Vasc Biol 27: 2470-2475, 2007.

13. Carmichael ST: Rodent models of focal stroke: size, mechanism, and purpose. NeuroRx 2: 396-409, 2005.
14. Liu H, Liu X, Wei X, et al: Losartan, an angiotensin II type 1 receptor blocker, ameliorates cerebral ischemia-reperfusion injury via PI3K/Akt-mediated enos phosphorylation. Brain Res Bull 89: 65-70, 2012.

15. Bradford MM: A rapid and sensitive method for quantitation of microgram quantities of protein utilizing the principle of protein-dye binding. Anal Biochem 72: 248-254, 1976.

16. Lu C, Liu L, Chen Y, et al: TLR2 ligand induces protection against cerebral ischemia/reperfusion injury via activation of phosphoinositide 3-kinase/Akt signaling. J Immunol 187: 1458-1466, 2011.

17. Shioda N, Ishigami T, Han F, et al: Activation of phosphatidylinositol 3-kinase/protein kinase B pathway by a vanadyl compound mediates its neuroprotective effect in mouse brain ischemia. Neuroscience 148: 221-229, 2007.

18. Soshnikova N, Zechner D, Huelsken J, et al: Genetic interaction between Wnt/beta-catenin and BMP receptor signaling during formation of the AER and the dorsal-ventral axis in the limb. Genes Dev 17: 1963-1968, 2003.

19. Wang J and Wynshaw-Boris A: The canonical Wnt pathway in early mammalian embryogenesis and stem cell maintenance/differentiation. Curr Opin Genet Dev 14: 533-539, 2004.

20. Lickert H, Domon C, Huls G, et al: Wnt/(beta)-catenin signaling regulates the expression of the homeobox gene $\mathrm{Cdx} 1$ in embryonic intestine. Development 127: 3805-3813, 2000.

21. MongaSP,Pediaditakis P,Mule K,StolzDB and Michalopoulos GK: Changes in WNT/beta-catenin pathway during regulated growth in rat liver regeneration. Hepatology 33: 1098-1109, 2001.

22. Reya $T$ and Clevers $H$ : Wnt signalling in stem cells and cancer. Nature 434: 843-850, 2005.

23. Kamada H, Nito C, Endo H and Chan PH: Bad as a converging signaling molecule between survival PI3-K/Akt and death JNK in neurons after transient focal cerebral ischemia in rats. J Cereb Blood Flow Metab 27: 521-533, 2007

24. Sun B, Chen L, Wei X, Xiang Y, Liu X and Zhang X: The Akt/GSK-3 $\beta$ pathway mediates flurbiprofen-induced neuroprotection against focal cerebral ischemia/reperfusion injury in rats. Biochem Biophys Res Commun 409: 808-813, 2011.

25. Chai YS, Hu J, Lei F, et al: Effect of berberine on cell cycle arrest and cell survival during cerebral ischemia and reperfusion and correlations with p53/cyclin D1 and PI3K/Akt. Eur J Pharmacol 708: 44-55, 2013.

26. Zhan L,LiD,Liang D, etal: Activation of Akt/FoxO and inactivation of MEK/ERK pathways contribute to induction of neuroprotection against transient global cerebral ischemia by delayed hypoxic postconditioning in adult rats. Neuropharmacology 63: 873-882, 2012.

27. Zhang J, Deng Z, Liao J, et al: Leptin attenuates cerebral ischemia injury through the promotion of energy metabolism via the PI3K/Akt pathway. J Cereb Blood Flow Metab 33: 567-574, 2013.

28. Ma YP, Ma MM, Cheng SM, et al: Intranasal bFGF-induced progenitor cell proliferation and neuroprotection after transient focal cerebral ischemia. Neurosci Lett 437: 93-97, 2008.

29. Maric D, Fiorio Pla A, Chang YH and Barker JL: Self-renewing and differentiating properties of cortical neural stem cells are selectively regulated by basic fibroblast growth factor (BFGF) signaling via specific FGF receptors. J Neurosci 27: 1836-1852, 2007.

30. Xing Y, Zhang X, Zhao K, et al: Beneficial effects of sulindac in focal cerebral ischemia: a positive role in $\mathrm{Wnt} / \beta$-catenin pathway. Brain Res 1482: 71-80, 2012.

31. Cai L, Ye Z, Zhou BY, Mali P, Zhou C and Cheng L: Promoting human embryonic stem cell renewal or differentiation by modulating Wnt signal and culture conditions. Cell Res 17: 62-72, 2007.

32. Endo H, Nito C, Kamada H, Nishi T and Chan PH: Activation of the Akt/GSK3beta signaling pathway mediates survival of vulnerable hippocampal neurons after transient global cerebral ischemia in rats. J Cereb Blood Flow Metab 26: 1479-1489, 2006.

33. Spaccapelo L, Galantucci M, Neri L, et al: Up-regulation of the canonical Wnt-3A and sonic hedgehog signaling underlies melanocortin-induced neurogenesis after cerebral ischemia. Eur J Pharmacol 707: 78-86, 2013. 